\title{
A NEW CLASS OF FUSION RULES BASED ON T-CONORM AND T-NORM FUZZY OPERATORS
}

\author{
Albena TCHAMOVA, Jean DEZERT and Florentin SMARANDACHE
}

\begin{abstract}
In this paper a particular combination rule based on specified fuzzy TConorm/T-Norm operators is proposed and analysed - TCN Rule of Combination. It does not belong to the general Weighted Operator Class. The nice features of the new rule could be defined as: very easy to implement, satisfying the impact of neutrality of Vacuous Belief Assignment; commutative, convergent to idempotence, reflecting majority opinion, assuring an adequate data processing in case of total conflict. Several numerical examples and comparisons with the new advanced Proportional Conflict Redistribution Rules proposed recently by Florentin Smarandache and Jean Dezert within their theory of plausible and paradoxical reasoning are presented.
\end{abstract}

Keywords: Information Fusion, Combination rules, Conjunctive rule, Fuzzy operators, Dezert-Smarandache theory (DSmT), Proportional Conflict Redistribution Rules.

\section{Introduction}

There are many combination rules available for information fusion $[5,8,7]$. No one of them can satisfy the whole range of requirements, associated with the all possible applications. In temporal multiple target tracking the main requirements they have to deal with relate especially to the way of adequate conflict processing/redistribution, the simplicity of implementation, satisfaction of the impact of neutrality of Vacuous Belief Assignment (VBA), reflection of majority opinion, etc. In this work we propose to connect the combination rules for information fusion with particular fuzzy operators: the Conjunctive rule is replaced with fuzzy T-norm operator and respectively the Disjunctive rule with T-conorm operator. These rules take their source from the T-norm and T-conorm operators in fuzzy logics, where the AND logic operator corresponds in information fusion to the conjunctive rule and the OR logic operator corresponds to the disjunctive rule. While the logic operators deal with degrees of truth and false, the fusion rules deal with degrees of belief of hypotheses. Within this work we will focus only on the T-norm based Conjunctive rule as an analog of the ordinary conjunctive

INFORMATION \& SECURITY. An International Journal, Vol. 20, 2006, 77-93 
rule of combination. It is because especially the conjunctive rule is appropriate for identification problems, restricting the set of hypotheses we are looking for.

\section{Fuzzy Inference for Information Fusion}

The main purpose of information fusion is to produce reasonably aggregated, refined and/or completed granule of data obtained from a single or multiple sources with consequent reasoning process. It means, that the main problem here consists in the way to aggregate correctly these sources of information, which in general case are imprecise, uncertain, or/and conflicting. Actually, there is no a single, unique rule to deal simultaneously with such kind of information peculiarities. Something more, there are a huge number of possible combinational rules, appropriate for a particular only application conditions. In [6] an unification of fusion theories and a combination of fusion rules in solving different problems is proposed. The most appropriate model for each considered application is selected. Here we will consider the case with a given Shafer's model $[4,1]$. Let $\Theta=\left\{\theta_{1}, \theta_{2}, \ldots \theta_{n}\right\}$ be the frame of discernment for the problem under consideration, where $\theta_{1}, \theta_{2}, \ldots \theta_{n}$ are a set of $\mathrm{n}$ exhaustive and exclusive hypotheses. Within the applied model, Dempster-Shafer's Power Set is described as: $2^{\theta}=\left\{\emptyset, \theta_{1}, \theta_{2}, \theta_{1} \cup \theta_{2}\right\}$. The basic belief assignment (bba) $m():. 2^{\Theta} \rightarrow[0,1]$, associated with a given information granule is defined with:

$$
m(\oslash)=0 ; \quad \sum_{X \in 2^{\ominus}} m(X)=1 .
$$

Having given two basic belief assignments $m_{1}($.$) and m_{2}($.$) and Shafer's model,$ Dempster's rule of combination [1] appears to be the most frequently used combination rule. It is defined as:

$$
m_{12}(X)=\frac{\sum_{\substack{x_{i} \cap X_{j}=X \\ x_{i}, x_{j} \in 2^{\Theta}}} m_{1}\left(X_{i}\right) \cdot m_{2}\left(X_{j}\right)}{1-\sum_{\substack{x_{i} \cap x_{j}=\varnothing \\ x_{i}, X_{j} \in 2^{\Theta}}} m_{1}\left(X_{i}\right) \cdot m_{2}\left(X_{j}\right)}
$$

The term $k=\sum_{\substack{x_{i} \cap x_{j}=\ominus \\ x_{i}, x_{j} \in 2^{\Theta}}} m_{1}\left(X_{i}\right) \cdot m_{2}\left(X_{j}\right)$ defines the degree of conflict between the sources of information. The normalization step (i.e. the division by $1-k$ ) in Dempster's rule is definitely the most sensitive and week point of the rule, because the fused result becomes a proper information granule only in the cases, when $k<1$. The new advanced Proportional Conflict Redistribution rules, proposed recently by F. Smarandache and J. Dezert [8], which are particular cases of the Weighted Operator overcome successfully the main limitations of Dempster's rule.

In this work our goal is to propose a new, alternative combination rule, interpreting the fusion in terms of fuzzy operators, which avoids the Dempster's rule weakness, possesses an adequate behavior in cases of total conflict and has an easy implementation. 


\subsection{The Way of Interpreting the Fusion}

We assume the relation between the two basic belief assignments (the information granules) $m_{1}($.$) and m_{2}($.$) to be considered as a vague relation, characterized with the$ following two characteristics:

* The way of association between the focal elements, included in the basic belief assignments of the sources of information. It is a particular operation chosen among the operations: union and intersection respectively. These set operations corresponds to logic operations Conjunction and Disjunction.

* The degree of association (interaction) between the focal elements included in the basic belief assignments of the sources of information. It is obtained as a Tnorm (for Conjunction) or T-conorm (for Disjunction) operators applied over the probability masses of corresponding focal elements. There are multiple choices available in order to define T-norm and T-conorm operators.

Within this work, as it is mentioned above, we will focus only on the T-norm based Conjunctive rule, more precisely Minimum T-norm based Conjunctive rule as an an$\log$ of the ordinary conjunctive rule of combination. We will demonstrate that it yields results very closed to conjunctive rule, satisfying the principle of neutrality of VBA, reflecting the majority opinion, converging towards idempotence and having adequate behavior in cases of total conflict presence. It is commutative, simply to apply, but not associative.

\subsection{Main properties of T-Norm Function}

The $T-$ norm $:[0,1]^{2} \mapsto[0,1]$ is a function defined in fuzzy set/logic theory in order to represent the intersection between two particular fuzzy sets and the AND fuzzy logical operator respectively. If one extends T-norm to the data fusion theory, it will be a substitute for the conjunctive rule. The T-norm has to satisfy the following conditions:

* Associativity:Tnorm $(\operatorname{Tnorm}(x, y), z)=\operatorname{Tnorm}(x, \operatorname{Tnorm}(y, z))$

* Commutativity: $\operatorname{Tnorm}(x, y)=\operatorname{Tnorm}(y, x)$

* Monotonicity: if $(x \leq a) \&(y \leq b)$ then Tnorm $(x, y) \leq \operatorname{Tnorm}(a, b)$

* Boundary Conditions: Tnorm $(0,0)=0 ; \operatorname{Tnorm}(x, 1)=x$

\subsection{Functions satisfying the $T$ - norm conditions}

There are many functions which satisfy the T-norm conditions:

* Zadeh's (default) min operator [3]: $m(X)=\min \left\{m_{1}\left(X_{i}\right), m_{2}\left(X_{j}\right)\right\}$ 
* Algebraic product operator: $m(X)=m_{1}\left(X_{i}\right) \cdot m_{2}\left(X_{j}\right)$

* Bounded product operator: $m(X)=\max \left\{\left[m_{1}\left(X_{i}\right)+m_{2}\left(X_{j}\right)\right], 0\right\}$

We are interested the chosen T-norm operator to satisfy the neutrality of VBA. From the described above functions, the default (min) and the algebraic product operator satisfy this condition. Taking it in mind we choose the default Minimum T-norm operator in order to define the degree of associations between the focal elements of information granules.

\subsection{Proving of the Vague min Set Operator}

The intersection $X_{i} \cap X_{j}$ for crisp (ordinary) subsets of the universe $U$ includes all of the elements in $X_{i}$ and $X_{j}$ :

$$
\begin{gathered}
m(X)=1, \quad \text { if } X \in X_{i} \text { and } X \in X_{j} \\
m(X)=0, \quad \text { if } X \notin X_{i} \text { or } X \notin X_{j}
\end{gathered}
$$

Let $X_{i}$ and $X_{j}$ are some vague subsets of $U$. What do mean the conditions from above for the case of intersection $X_{i} \cap X_{j}$ :

* First condition $X \in X_{i}$ and $X \in X_{j}$

It means that the following case exists: $\left\{m\left(X \in X_{i}\right)=1, m\left(X \in X_{j}\right)=1\right\}$, for which: $\min \left\{m\left(X \in X_{i}\right), m\left(X \in X_{j}\right)\right\}=1$

* Second condition $X \notin X_{i}$ or $X \notin X_{j}$

It means that one of the following cases exist:

$$
\begin{aligned}
& \left\{m\left(X \in X_{i}\right)=0, m\left(X \in X_{j}\right)=0\right\} \text { or } \\
& \left\{m\left(X \in X_{i}\right)=1, m\left(X \in X_{j}\right)=0\right\} \text { or } \\
& \left\{m\left(X \in X_{i}\right)=0, m\left(X \in X_{j}\right)=1\right\}
\end{aligned}
$$

for which: $\min \left\{m\left(X \in X_{i}\right), m\left(X \in X_{j}\right)\right\}=0$

From these it follows that $\min \left\{m\left(X \in X_{i}\right), m\left(X \in X_{j}\right)\right\}$ provides the correct expression for intersection.

\section{The T-conorm/T-norm (TCN) Rule of Combination}

Let's take a look at a general form of a fusion Table 1, where the T-norm based interpretation of the ordinary conjunctive rule of combination is considered for two given sources. The frame of the fusion problem under consideration is $\Theta=\left\{\theta_{1}, \theta_{2}\right\}$ and the power set is: $2^{\theta}=\left\{\emptyset, \theta_{1}, \theta_{2}, \theta_{1} \cup \theta_{2}\right\}$. The two basic belief assignments (sources of information) $m_{1}($.$) and m_{2}($.$) are defined over 2^{\Theta}$. It is assumed that $m_{1}($.$) and m_{2}($. are normalized bbas $\left(m(\oslash)=0 ; \quad \sum_{X \in 2^{\Theta}} m(X)=1\right)$. 
Step 1: Defining the min T-norm conjunctive consensus:

The min T-norm conjunctive consensus is based on the default min T-norm function. The way of association between the focal elements of the given two sources of information is defined as $X=X_{i} \cap X_{j}$, and the degree of association is as follows:

$$
\tilde{m}(X)=\min \left\{m_{1}\left(X_{i}\right), m_{2}\left(X_{j}\right)\right\},
$$

where $\tilde{m}(X)$ represents $^{1}$ the mass of belief associated to the given proposition $X$ by using T-Norm based conjunctive rule .

\begin{tabular}{|c|c|c|c|}
\hline & $m_{2}\left(\theta_{1}\right)$ & $m_{2}\left(\theta_{2}\right)$ & $m_{2}\left(\theta_{1} \cup \theta_{2}\right)$ \\
\hline$m_{1}\left(\theta_{1}\right)$ & $\begin{array}{c}\theta_{1} \cap \theta_{1}=\theta_{1} \\
\tilde{m}\left(\theta_{1}\right)= \\
\min \left\{m_{1}\left(\theta_{1}\right), m_{2}\left(\theta_{1}\right)\right\}\end{array}$ & $\begin{array}{c}\theta_{1} \cap \theta_{2} \\
\tilde{m}\left(\theta_{1} \cap \theta_{2}\right)= \\
\min \left\{m_{1}\left(\theta_{1}\right), m_{2}\left(\theta_{2}\right)\right\}\end{array}$ & $\begin{array}{c}\theta_{1} \cap\left(\theta_{1} \cup \theta_{2}\right)=\theta_{1} \\
\tilde{m}\left(\theta_{1}\right)= \\
\min \left\{m_{1}\left(\theta_{1}\right), m_{2}\left(\theta_{1} \cup \theta_{2}\right)\right\}\end{array}$ \\
\hline$m_{1}\left(\theta_{2}\right)$ & $\begin{array}{c}\left(\theta_{1} \cap \theta_{2}\right) \\
\tilde{m}\left(\theta_{1} \cap \theta_{2}\right)= \\
\min \left\{m_{1}\left(\theta_{2}\right), m_{2}\left(\theta_{1}\right)\right\}\end{array}$ & $\begin{array}{c}\theta_{2} \cap \theta_{2}=\theta_{2} \\
\tilde{m}\left(\theta_{2}\right)= \\
\min \left\{m_{1}\left(\theta_{2}\right), m_{2}\left(\theta_{2}\right)\right\}\end{array}$ & $\begin{array}{c}\theta_{2} \cap\left(\theta_{1} \cup \theta_{2}\right)=\theta_{2} \\
\tilde{m}\left(\theta_{2}\right)= \\
\min \left\{m_{1}\left(\theta_{2}\right), m_{2}\left(\theta_{1} \cup \theta_{2}\right)\right\}\end{array}$ \\
\hline$m_{1}\left(\theta_{1} \cup \theta_{2}\right)$ & $\begin{array}{c}\left(\theta_{1} \cup \theta_{2}\right) \cap \theta_{1}=\theta_{1} \\
\tilde{m}\left(\theta_{1}\right)= \\
\min \left\{m_{1}\left(\theta_{1} \cup \theta_{2}\right), m_{2}\left(\theta_{1}\right)\right\}\end{array}$ & $\begin{array}{c}\left(\theta_{1} \cup \theta_{2}\right) \cap \theta_{2}=\theta_{2} \\
\tilde{m}\left(\theta_{2}\right)= \\
\min \left\{m_{1}\left(\theta_{1} \cup \theta_{2}\right), m_{2}\left(\theta_{2}\right)\right\}\end{array}$ & $\begin{array}{c}\left(\theta_{1} \cup \theta_{2}\right) \cap\left(\theta_{1} \cup \theta_{2}\right)=\theta_{1} \cup \theta_{2} \\
\tilde{m}\left(\theta_{1} \cup \theta_{2}\right)= \\
\min \left\{m_{1}\left(\theta_{1} \cup \theta_{2}\right), m_{2}\left(\theta_{1} \cup \theta_{2}\right)\right\}\end{array}$ \\
\hline
\end{tabular}

Table 1:Min T-norm based Interpretation of Conjunctive Rule

The proposed T-conorm/T-norm based Combination rule, called by the authors TCN rule of combination, in Dempster Shafer Theory framework is defined for $\forall X \in$ $2^{\Theta}$ by the equation:

$$
\tilde{m}(X)=\sum_{\substack{x_{i} \cap X_{j}=x \\ x_{i}, x_{j} \in 2^{\Theta}}} \min \left\{m_{1}\left(X_{i}\right), m_{2}\left(X_{j}\right)\right\}
$$

Step 2: Distribution of the mass, assigned to the conflict

The distribution of the mass, assigned to the conflict follows in some degree the distribution of conflicting mass in DSmT Proportional Conflict Redistribution Rule 2 [8], but the procedure here is based on fuzzy operators. Let us denote the two bbas, associated with the information sources in a matrix form:

$$
\left[\begin{array}{l}
m_{1}(.) \\
m_{2}(.)
\end{array}\right]=\left[\begin{array}{lll}
m_{1}\left(\theta_{1}\right) & m_{1}\left(\theta_{2}\right) & m_{1}\left(\theta_{1} \cup \theta_{2}\right) \\
m_{2}\left(\theta_{1}\right) & m_{2}\left(\theta_{2}\right) & m_{2}\left(\theta_{1} \cup \theta_{2}\right)
\end{array}\right]
$$

The total conflicting mass is distributed to all non-empty sets proportionally with respect to the maximum, (denoted here as $x_{12}(X)$ ) between the elements of corresponding mass matrix's columns, associated with the element $X$ of the power set. It means

\footnotetext{
${ }^{1}$ We introduce in this paper the over-tilded notation for masses to specify that the masses of belief are obtained with fuzzy T-norm and T-conorm operators.
} 
the bigger mass is redistributed towards the element, involved in the conflict and contributing to the conflict with the maximum specified probability mass. The fuzzy operator maximum is used in order to interpret the summation of corresponding mass matrix's columns, associated with the element $X$ of the power set, as used in DSmT Proportional Conflict Redistribution Rules.

$$
\begin{aligned}
& x_{12}\left(\theta_{1}\right)=\max \left(m_{1}\left(\theta_{1}\right), m_{2}\left(\theta_{1}\right)\right) \\
& x_{12}\left(\theta_{2}\right)=\max \left(m_{1}\left(\theta_{2}\right), m_{2}\left(\theta_{2}\right)\right)
\end{aligned}
$$

One denotes by $r\left(\theta_{1}\right)$ and $r\left(\theta_{2}\right)$ the part of conflicting mass, distributed to the propositions $\theta_{1}$ and $\theta_{2}$. Then one has:

$$
\frac{r\left(\theta_{1}\right)}{x_{12}\left(\theta_{1}\right)}=\frac{r\left(\theta_{2}\right)}{x_{12}\left(\theta_{2}\right)}=\frac{r\left(\theta_{1}\right)+r\left(\theta_{2}\right)}{x_{12}\left(\theta_{1}\right)+x_{12}\left(\theta_{2}\right)}=\frac{k_{12}}{s_{12}}
$$

Then, the conflicting masses that have to be redistributed are:

$$
r\left(\theta_{1}\right)=x_{12}\left(\theta_{1}\right) \cdot \frac{k_{12}}{s_{12}} ; r\left(\theta_{2}\right)=x_{12}\left(\theta_{2}\right) \cdot \frac{k_{12}}{s_{12}} .
$$

Finally the bba obtained as a result of the applied TCN rule with fuzzy based Proportional Conflict Redistribution Rule 2 , denoted here as $\tilde{m}_{P C R 2}($.$) becomes:$

$$
\begin{gathered}
\tilde{m}_{P C R 2}\left(\theta_{1}\right)=\tilde{m}\left(\theta_{1}\right)+x_{12}\left(\theta_{1}\right) \cdot \frac{k_{12}}{s_{12}} \\
\tilde{m}_{P C R 2}\left(\theta_{2}\right)=\tilde{m}\left(\theta_{2}\right)+x_{12}\left(\theta_{2}\right) \cdot \frac{k_{12}}{s_{12}} \\
\tilde{m}_{P C R 2}\left(\theta_{1} \cup \theta_{2}\right)=\tilde{m}\left(\theta_{1} \cup \theta_{2}\right),
\end{gathered}
$$

where $k_{12}$ is the total conflict;

$$
x_{12}(X)=\max _{i=1,2}\left(m_{i}(X)\right) \neq 0
$$

and $s_{12}$ is the sum of all non-zero maximum values of column's masses, assigned to non-empty sets. The conflict mass is redistributed only to the propositions, involved in the conflict.

Step 3: Normalization of the result: The final step of the TCN rule concerns the normalization procedure:

$$
\tilde{m}_{P C R 2}(X)=\frac{\tilde{m}_{P C R 2}(X)}{\sum_{\substack{x \neq 0 \\ X \in 2^{\Theta}}} \tilde{m}_{P C R 2}(X)}
$$




\section{Implementation of the TCN Rule of Combination}

\subsection{Example 1}

Let's have the frame of the problem $\theta=\left\{\theta_{1}, \theta_{2}\right\}$ and two independent sources of information with basic belief assignments, as follows:

$$
\begin{array}{lll}
m_{1}\left(\theta_{1}\right)=0.6 & m_{1}\left(\theta_{2}\right)=0.2 & m_{1}\left(\theta_{1} \cup \theta_{2}\right)=0.2 \\
m_{2}\left(\theta_{1}\right)=0.4 & m_{2}\left(\theta_{2}\right)=0.5 & m_{5}\left(\theta_{1} \cup \theta_{2}\right)=0.1
\end{array}
$$

The min T-norm based conjunctive consensus yields here (Table 2):

\begin{tabular}{|c|c|c|c|}
\hline & $m_{2}\left(\theta_{1}\right)=0.4$ & $m_{2}\left(\theta_{2}\right)=0.5$ & $m_{2}\left(\theta_{1} \cup \theta_{2}\right)=0.1$ \\
\hline$m_{1}\left(\theta_{1}\right)=0.6$ & $\begin{array}{c}\tilde{m}\left(\theta_{1}\right)= \\
\min (0.6,0.4)=0.4\end{array}$ & $\begin{array}{c}\tilde{m}\left(\theta_{1} \cap \theta_{2}\right)= \\
\min (0.6,0.5)=0.5\end{array}$ & $\begin{array}{c}\tilde{m}\left(\theta_{1}\right)= \\
\min (0.6,0.1)=0.1\end{array}$ \\
\hline$m_{1}\left(\theta_{2}\right)=0.2$ & $\begin{array}{c}\tilde{m}\left(\theta_{1} \cap \theta_{2}\right)= \\
\min (0.2,0.4)=0.2\end{array}$ & $\begin{array}{c}\tilde{m}\left(\theta_{2}\right)= \\
\min (0.2,0.5)=0.2\end{array}$ & $\begin{array}{c}\tilde{m}\left(\theta_{2}\right)= \\
\min (0.2,0.1)=0.1\end{array}$ \\
\hline$m_{1}\left(\theta_{1} \cup \theta_{2}\right)=0.2$ & $\begin{array}{c}\tilde{m}\left(\theta_{1}\right)= \\
\min (0.2,0.4)=0.2\end{array}$ & $\begin{array}{c}\tilde{m}\left(\theta_{2}\right)= \\
\min (0.2,0.5)=0.2\end{array}$ & $\begin{array}{c}\tilde{m}\left(\theta_{1} \cup \theta_{2}\right)= \\
\min (0.2,0.1)=0.1\end{array}$ \\
\hline
\end{tabular}

Table 2: Min T-norm based Interpretation of Conjunctive Rule

\subsubsection{Fusion with TCN Rule of Combination}

Step 1: Obtaining min T-norm Conjunctive Consensus

Using Table 2 and applying equation 1, the fusion result becomes:

$$
\begin{gathered}
\tilde{m}\left(\theta_{1}\right)=0.4+0.2+0.1=0.7 \\
\tilde{m}\left(\theta_{2}\right)=0.2+0.1+0.2=0.5 \\
\tilde{m}\left(\theta_{1} \cap \theta_{2}\right)=0.5+0.2=0.7 \\
\tilde{m}\left(\theta_{1} \cup \theta_{2}\right)=0.1
\end{gathered}
$$

Step 2: Redistribution of the conflict by using fuzzy based PCR2

$$
\begin{aligned}
\frac{r\left(\theta_{1}\right)}{\max \left(m_{1}\left(\theta_{1}\right),\left(m_{2}\left(\theta_{1}\right)\right)\right.} & =\frac{r\left(\theta_{2}\right)}{\max \left(m_{1}\left(\theta_{2}\right),\left(m_{2}\left(\theta_{2}\right)\right)\right.}= \\
\frac{r\left(\theta_{1}\right)}{\max (0.6,0.4)} & =\frac{r\left(\theta_{2}\right)}{\max (0.2,0.5)}=
\end{aligned}
$$




$$
\begin{aligned}
& \frac{r\left(\theta_{1}\right)+r\left(\theta_{2}\right)}{\max (0.6,0.4)+\max (0.2,0.5)}=\frac{\tilde{m}\left(\theta_{1} \cap \theta_{2}\right)}{0.6+0.5}=\frac{0.7}{1.1}=0.636 \\
& r\left(\theta_{1}\right)=0.6 \cdot 0.636=0.3816 ; \quad r\left(\theta_{2}\right)=0.5 \cdot 0.636=0.318 ;
\end{aligned}
$$

Then, after the conflict redistribution, the new masses become:

$$
\begin{gathered}
\tilde{m}_{P C R 2}(.)=\left\{\tilde{m}_{P C R 2}\left(\theta_{1}\right)=0.7+0.3816=1.0816 ;\right. \\
\tilde{m}_{P C R 2}\left(\theta_{2}\right)=0.5+0.318=0.818, \\
\left.\tilde{m}_{P C R 2}\left(\theta_{1} \cup \theta_{2}\right)=0.1\right\}
\end{gathered}
$$

Step 3: Normalization of the result

After the normalization procedure one gets the final information granule, as follows:

$\tilde{m}_{P C R 2}()=.\left\{\tilde{m}_{P C R 2}\left(\theta_{1}\right)=0.54, \tilde{m}_{P C R 2}\left(\theta_{2}\right)=0.41, \tilde{m}_{P C R 2}\left(\theta_{1} \cup \theta_{2}\right)=0.05\right\}$

\subsubsection{Fusion with Ordinary Conjunctive Rule}

The conjunctive consensus here is given by:

$$
m\left(\theta_{1}\right)=0.38, m\left(\theta_{2}\right)=0.22, m\left(\theta_{1} \cap \theta_{2}\right)=k=0.38 m\left(\theta_{1} \cup \theta_{2}\right)=0.02
$$

The PCR2 rule $[7,8]$ is used in order to redistribute the obtained conflict:

$$
\frac{x}{0.4+0.6}=\frac{y}{0.5+0.2}=\frac{x+y}{1.7}=\frac{0.38}{1.7}=0.224
$$

Then, the final masses of belief become:

$$
\begin{gathered}
m_{P C R 2}\left(\theta_{1}\right)=0.38+1.0 \cdot 0.224=0.604 \\
m_{P C R 2}\left(\theta_{2}\right)=0.22+0.7 \cdot 0.224=0.376 \\
m_{P C R 2}\left(\theta_{1} \cup \theta_{2}\right)=0.02
\end{gathered}
$$

\begin{tabular}{|c|c|}
\hline Ordinary Conjunctive Rule with PCR2 & TCN Rule with fuzzy based PCR2 \\
\hline$m_{P C R 2}\left(\theta_{1}\right)=0.604$ & $\tilde{m}_{P C R 2}\left(\theta_{1}\right)=0.54$ \\
\hline$m_{P C R 2}\left(\theta_{2}\right)=0.376$ & $\tilde{m}_{P C R 2}\left(\theta_{1}\right)=0.41$ \\
\hline$m_{P C R 2}\left(\theta_{1} \cup \theta_{2}\right)=0.02$ & $\tilde{m}_{P C R 2}\left(\theta_{1} \cup \theta_{2}\right)=0.05$ \\
\hline
\end{tabular}

Table 3: Comparative Results

In the Table 3 there are given comparative results obtained by using ordinary conjunctive rule with PCR2 redistribution of conflicting mass and TCN rule with fuzzy based PCR2. 


\subsection{Zadeh's Example}

Let's have $\theta=\left\{\theta_{1}, \theta_{2}, \theta_{3}\right\}$ and two independent sources of information with the corresponding bbas $[9,10]$ :

$$
\begin{array}{lll}
m_{1}\left(\theta_{1}\right)=0.99 & m_{1}\left(\theta_{2}\right)=0.0 & m_{1}\left(\theta_{3}\right)=0.01 \\
m_{2}\left(\theta_{1}\right)=0.0 & m_{2}\left(\theta_{2}\right)=0.99 & m_{2}\left(\theta_{3}\right)=0.01
\end{array}
$$

4.2.1 Fusion with TCN Rule of Combination

Here the min T-norm based conjunctive consensus yield the following result:

$\tilde{m}\left(\theta_{3}\right)=0.01 ; \quad \tilde{m}\left(\theta_{1} \cap \theta_{2}\right)=0.99 ; \quad \tilde{m}\left(\theta_{1} \cap \theta_{3}\right)=0.01 ; \quad \tilde{m}\left(\theta_{2} \cap \theta_{3}\right)=0.01 ;$

The partial conflicting masses will be redistributed to corresponding non-empty sets, contributing to the particular partial conflicts by using fuzzy based PCR3. According to $\tilde{m}\left(\theta_{1} \cap \theta_{2}\right)=0.99$ :

$$
\begin{gathered}
\frac{x_{1}}{\max (0,0.99)}=\frac{y_{1}}{\max (0,0.99)}=\frac{x_{1}+y_{1}}{1.98}=\frac{0.99}{1.98}=0.5 \\
x_{1}=0.99 \cdot 0.5=0.495 ; \quad y_{1}=0.99 \cdot 0.5=0.495
\end{gathered}
$$

According to $\tilde{m}\left(\theta_{1} \cap \theta_{3}\right)=0.01$ :

$$
\begin{gathered}
\frac{x_{2}}{\max (0,0.99)}=\frac{z_{1}}{\max (0.01,0.01)}=\frac{x_{2}+z_{1}}{1.0}=\frac{0.01}{1.0}=0.01 \\
x_{2}=0.99 \cdot 0.01=0.0099 ; \quad z_{1}=0.01 \cdot 0.01=0.0001
\end{gathered}
$$

According to $\tilde{m}\left(\theta_{2} \cap \theta_{3}\right)=0.01$ :

$$
\begin{gathered}
\frac{y_{2}}{\max (0,0.99)}=\frac{z_{2}}{\max (0.01,0.01)}=\frac{y_{2}+z_{2}}{1.0}=\frac{0.01}{1.0}=0.01 \\
y_{2}=0.99 \cdot 0.01=0.0099 ; \quad z_{2}=0.01 \cdot 0.01=0.0001
\end{gathered}
$$

After the conflict redistribution by using fuzzy based PCR3, the result obtained becomes:

$$
\begin{gathered}
\tilde{m}_{P C R 3}\left(\theta_{1}\right)=\tilde{m}\left(\theta_{1}\right)+x_{1}+x_{2}=0+0.495+0.0099=0.5049 \\
\tilde{m}_{P C R 3}\left(\theta_{2}\right)=\tilde{m}\left(\theta_{2}\right)+y_{1}+y_{2}=0+0.495+0.0099=0.5049 \\
\tilde{m}_{P C R 3}\left(\theta_{3}\right)=\tilde{m}\left(\theta_{3}\right)+z_{1}+z_{2}=0.01+0.0001+0.0001=0.0102
\end{gathered}
$$

The normalization leads to the following final result:

$\tilde{m}_{P C R 3}()=.\left\{\tilde{m}_{P C R 3}\left(\theta_{1}\right)=0.495, \quad \tilde{m}_{P C R 3}\left(\theta_{2}\right)=0.495, \quad \tilde{m}_{P C R 3}\left(\theta_{3}\right)=0.01\right\}$ 


\subsubsection{Fusion with Ordinary Conjunctive Rule}

The conjunctive consensus is given by:

$m\left(\theta_{3}\right)=0.0001 ; \quad m\left(\theta_{1} \cap \theta_{2}\right)=0.98 ; \quad m\left(\theta_{1} \cap \theta_{3}\right)=0.0099 ; \quad m\left(\theta_{2} \cap \theta_{3}\right)=0.0099$

Applying PCR3 $[7,8]$ rule to the partial conflicting masses, one gets:

According to $m\left(\theta_{1} \cap \theta_{2}\right)=0.98$ :

$$
\frac{x_{1}}{0.99+0.0}=\frac{y_{1}}{0.99+0.0}=\frac{x_{1}+y_{1}}{1.98}=\frac{0.98}{1.98}=0.495
$$

According to $m\left(\theta_{1} \cap \theta_{3}\right)=0.0099$ :

$$
\frac{x_{2}}{0.99}=\frac{z_{1}}{0.02}=\frac{x_{2}+z_{1}}{1.01}=\frac{0.0099}{1.01}=0.0098
$$

According to $m\left(\theta_{2} \cap \theta_{3}\right)=0.0099$ :

$$
\frac{y_{2}}{0.99}=\frac{z_{2}}{0.02}=\frac{y_{2}+z_{2}}{1.01}=\frac{0.0099}{1.01}=0.0098
$$

Finally, the result is given by:

$$
\begin{gathered}
m_{P C R 3}\left(\theta_{1}\right)=0+(0.99 \cdot 0.495)+(0.99 \cdot 0.0098)=0.49975 \\
m_{P C R 3}\left(\theta_{2}\right)=0+(0.99 \cdot 0.495)+(0.99 \cdot 0.0098)=0.49975 \\
m_{P C R 3}\left(\theta_{3}\right)=0.0001+(0.02 \cdot 0.0098)+(0.02 \cdot 0.0098)=0.0005
\end{gathered}
$$

The comparative results are given in the Table 4 .

\begin{tabular}{|c|c|}
\hline Ordinary Conjunctive Rule with PCR3 & TCN Rule with fuzzy based PCR3 \\
\hline$m_{P C R 3}\left(\theta_{1}\right)=0.49975$ & $\tilde{m}_{P C R 3}\left(\theta_{1}\right)=0.495$ \\
\hline$m_{P C R 3}\left(\theta_{2}\right)=0.49975$ & $\tilde{m}_{P C R 3}\left(\theta_{2}\right)=0.495$ \\
\hline$m_{P C R 3}\left(\theta_{3}\right)=0.0005$ & $\tilde{m}_{P C R 3}\left(\theta_{3}\right)=0.01$ \\
\hline
\end{tabular}

Table 4: Comparative Results 


\subsection{Total Conflict Example}

Let's consider a case with the frame of the problem $\theta=\left\{\theta_{1}, \theta_{2}, \theta_{3}, \theta_{4}\right\}$ and two independent sources of information:

$$
\begin{array}{llll}
m_{1}\left(\theta_{1}\right)=0.3 & m_{1}\left(\theta_{2}\right)=0.0 & m_{1}\left(\theta_{3}\right)=0.7 & m_{1}\left(\theta_{4}\right)=0.0 \\
m_{2}\left(\theta_{1}\right)=0.0 & m_{2}\left(\theta_{2}\right)=0.4 & m_{2}\left(\theta_{3}\right)=0.0 & m_{2}\left(\theta_{4}\right)=0.6
\end{array}
$$

4.3.1 Fusion with TCN Rule of Combination

Here the min T-norm conjunctive consensus yield the following result:

$$
\tilde{m}\left(\theta_{1} \cap \theta_{2}\right)=0.3 ; \tilde{m}\left(\theta_{1} \cap \theta_{4}\right)=0.3 ; \quad \tilde{m}\left(\theta_{2} \cap \theta_{3}\right)=0.4 ; \quad \tilde{m}\left(\theta_{3} \cap \theta_{4}\right)=0.6 ;
$$

Here one obtains the partial conflicting masses that will be redistributed by using fuzzy base PCR3.

According to the partial conflict $\tilde{m}\left(\theta_{1} \cap \theta_{2}\right)=0.3$ :

$$
\frac{x_{1}}{\max (0,0.3)}=\frac{y_{1}}{\max (0,0.4)}=\frac{x_{1}+y_{1}}{0.7}=\frac{0.3}{0.7}=0.4285
$$

According to the partial conflict $\tilde{m}\left(\theta_{1} \cap \theta_{4}\right)=0.3$ :

$$
\frac{x_{2}}{\max (0,0.3)}=\frac{h_{1}}{\max (0,0.6)}=\frac{x_{2}+h_{1}}{0.9}=\frac{0.3}{0.9}=0.3333
$$

According to the partial conflict $\tilde{m}\left(\theta_{2} \cap \theta_{3}\right)=0.4$ :

$$
\frac{y_{2}}{\max (0,0.4)}=\frac{z_{1}}{\max (0,0.7)}=\frac{y_{2}+z_{1}}{1.1}=\frac{0.4}{1.1}=0.3636
$$

According to the partial conflict $\tilde{m}\left(\theta_{3} \cap \theta_{4}\right)=0.6$ :

$$
\frac{z_{2}}{\max (0,0.7)}=\frac{h_{2}}{\max (0,0.6)}=\frac{z_{2}+h_{2}}{1.3}=\frac{0.6}{1.3}=0.4615
$$

After the conflict redistribution, the result is given by:

$$
\begin{array}{ll}
\tilde{m}_{P C R 3}\left(\theta_{1}\right)=0.2275 ; & \tilde{m}_{P C R 3}\left(\theta_{2}\right)=0.3168 ; \\
\tilde{m}_{P C R 3}\left(\theta_{3}\right)=0.5775 ; & \tilde{m}_{P C R 3}\left(\theta_{4}\right)=0.4768 ;
\end{array}
$$

The normalization procedure yields the following final result:

$$
\begin{aligned}
& \tilde{m}_{P C R 3}\left(\theta_{1}\right)=0.1423, \tilde{m}_{P C R 3}\left(\theta_{2}\right)=0.1982, \\
& \tilde{m}_{P C R 3}\left(\theta_{3}\right)=0.3612, \quad \tilde{m}_{P C R 3}\left(\theta_{4}\right)=0.2983 .
\end{aligned}
$$

\subsubsection{Fusion with Ordinary Conjunctive Rule}


The conjunctive consensus is given by:

$m\left(\theta_{1} \cap \theta_{2}\right)=0.12 ; \quad m\left(\theta_{1} \cap \theta_{4}\right)=0.18 ; \quad m\left(\theta_{2} \cap \theta_{3}\right)=0.28 ; \quad m\left(\theta_{3} \cap \theta_{4}\right)=0.42$

After applying PCR3 rule to the partial conflicting masses, finally one gets:

$$
\begin{aligned}
& m_{P C R 3}\left(\theta_{1}\right)=0.111 ; \quad m_{P C R 3}\left(\theta_{2}\right)=0.171 ; \\
& m_{P C R 3}\left(\theta_{3}\right)=0.404 ; \quad m_{P C R 3}\left(\theta_{4}\right)=0.314 ;
\end{aligned}
$$

The comparative results are given in the Table 5.

\begin{tabular}{|c|c|}
\hline Ordinary Conjunctive Rule with PCR3 & TCN Rule with fuzzy based PCR3 \\
\hline$m_{P C R 3}\left(\theta_{1}\right)=0.111$ & $\tilde{m}_{P C R 3}\left(\theta_{1}\right)=0.1423$ \\
\hline$m_{P C R 3}\left(\theta_{2}\right)=0.171$ & $\tilde{m}_{P C R 3}\left(\theta_{2}\right)=0.1982$ \\
\hline$m_{P C R 3}\left(\theta_{3}\right)=0.404$ & $\tilde{m}_{P C R 3}\left(\theta_{3}\right)=0.3612$ \\
\hline$m_{P C R 3}\left(\theta_{4}\right)=0.314$ & $\tilde{m}_{P C R 3}\left(\theta_{4}\right)=0.2983$ \\
\hline
\end{tabular}

Table 5: Comparative Results

\subsection{Example 5 (convergence to idempotence)}

Let's consider a case with the frame of the problem $\theta=\left\{\theta_{1}, \theta_{2}\right\}$ and two independent sources of information:

$$
\begin{array}{ll}
m_{1}(.)=\left\{m_{1}\left(\theta_{1}\right)=0.7 ;\right. & \left.m_{1}\left(\theta_{2}\right)=0.3\right\} \\
m_{2}(.)=\left\{m_{2}\left(\theta_{1}\right)=0.7 ;\right. & \left.m_{2}\left(\theta_{2}\right)=0.3\right\}
\end{array}
$$

4.4.1 Fusion with TCN Rule of Combination

Here the min T-norm conjunctive consensus yield the following result:

$$
\tilde{m}(.)=\left\{\tilde{m}\left(\theta_{1}\right)=0.7 ; \quad \tilde{m}\left(\theta_{2}\right)=0.3 ; \quad \tilde{m}\left(\theta_{1} \cap \theta_{2}\right)=0.6 ;\right\}
$$

After the conflict redistribution by using fuzzy based PCR2 one gets:

$$
\begin{aligned}
& \frac{x}{\max (0.7,0.7)}=\frac{y}{\max (0.3,0.3)}=\frac{0.6}{1.0}=0.6 \\
& \tilde{m}_{P C R 2}\left(\theta_{1}\right)=1.12 ; \quad \tilde{m}_{P C R 2}\left(\theta_{2}\right)=0.48 ;
\end{aligned}
$$


After normalization the final fused result becomes:

$$
\tilde{m}_{P C R 2}(.)=\left\{\tilde{m}_{P C R 2}\left(\theta_{1}\right)=0.7 ; \quad \tilde{m}_{P C R 2}\left(\theta_{2}\right)=0.3 ;\right\}
$$

\subsubsection{Fusion with Ordinary Conjunctive Rule}

The conjunctive consensus is given by:

$$
m\left(\theta_{1}\right)=0.49 ; \quad m\left(\theta_{2}\right)=0.09 ; \quad m\left(\theta_{1} \cap \theta_{2}\right)=0.42 ;
$$

Finally, the vector of belief masses, after applying the PCR2 rule to the partial conflicting mass becomes:

$$
m_{P C R 2}\left(\theta_{1}\right)=0.784 ; m_{P C R 2}\left(\theta_{2}\right)=0.216
$$

The comparative results are given in the Table 6.

\begin{tabular}{|c|c|}
\hline Ordinary Conjunctive Rule with PCR2 & TCN Rule with fuzzy based PCR2 \\
\hline$m_{P C R 2}\left(\theta_{1}\right)=0.784$ & $\tilde{m}_{P C R 2}\left(\theta_{1}\right)=0.7$ \\
\hline$m_{P C R 2}\left(\theta_{2}\right)=0.216$ & $\tilde{m}_{P C R 2}\left(\theta_{2}\right)=0.3$ \\
\hline
\end{tabular}

Table 6: Comparative Results

It is evident, the fusion results obtained by using TCN Rule of combination converges strongly towards idempotence.

\subsection{Example 6 (majority opinion)}

Let's consider a case with the frame of the problem $\theta=\left\{\theta_{1}, \theta_{2}\right\}$ and two independent sources of information:

$$
\begin{array}{ll}
m_{1}(.)=\left\{m_{1}\left(\theta_{1}\right)=0.8 ;\right. & \left.m_{1}\left(\theta_{2}\right)=0.2\right\} \\
m_{2}(.)=\left\{m_{2}\left(\theta_{1}\right)=0.3 ;\right. & \left.m_{2}\left(\theta_{2}\right)=0.7\right\}
\end{array}
$$

Assume that in the next time moment a third source of information is introduced with the following bba:

$$
m_{3}(.)=\left\{m_{3}\left(\theta_{1}\right)=0.3 ; \quad m_{3}\left(\theta_{2}\right)=0.7\right\}
$$

4.5.1 Fusion with TCN Rule of Combination

The TCN rule with fuzzy based PCR2 yield the following normalized fusion result:

$$
\tilde{m}_{12 P C R 2}\left(\theta_{1}\right)=0.557 ; \quad \tilde{m}_{12 P C R 2}\left(\theta_{2}\right)=0.443
$$


Let's now combine $\tilde{m}_{12 P C R 2}($.$) with the gbba of the third source m_{3}($.$) . Then the final$ fused result is obtained:

$$
\tilde{m}_{12,3 P C R 2}\left(\theta_{1}\right)=0.417 ; \quad \tilde{m}_{12,3 P C R 2}\left(\theta_{2}\right)=0.583
$$

From this result it is evident, that the final bba $\tilde{m}_{12,3 P C R 2}()=.\left[\begin{array}{ll}0.417 & 0.583\end{array}\right]$ starts to reflect the majority opinion, it means that $\tilde{m}_{12,3 P C R 2}\left(\theta_{1}\right)<\tilde{m}_{12,3 P C R 2}\left(\theta_{2}\right)$. If fourth source is considered with a probability mass vector, supporting the majority opinion, i.e. $m_{4}()=.\left\{m_{4}\left(\theta_{1}\right)=0.3 ; \quad m_{4}\left(\theta_{2}\right)=0.7\right\}$, then the final probability mass vector becomes:

$$
\tilde{m}_{(12,3), 4 P C R 2}\left(\theta_{1}\right)=0.348 ; \quad \tilde{m}_{(12,3), 4 P C R 2}\left(\theta_{2}\right)=0.652 ;
$$

The new fused vector $\tilde{m}_{(12,3), 4 P C R 2}()=.\left[\begin{array}{ll}0.348 & 0.652\end{array}\right]$ reflects again the majority opinion, since $\tilde{m}_{(12,3), 4 P C R 2}\left(\theta_{1}\right)$ decreases more and more and in the same time $\tilde{m}_{(12,3), 4 P C R 2}\left(\theta_{2}\right)$ increases in the same manner.

\subsubsection{Fusion with Ordinary Conjunctive Rule}

The conjunctive consensus between sources 1 and 2 is given by:

$$
m_{12}(.)=\left\{m_{12}\left(\theta_{1}\right)=0.24 ; \quad m_{12}\left(\theta_{2}\right)=0.14 ; \quad m_{12}\left(\theta_{1} \cap \theta_{2}\right)=0.62\right\}
$$

After applying the PCR2 rule to the partial conflicting mass $m_{12}\left(\theta_{1} \cap \theta_{2}\right)=0.62$, the final probability mass vector becomes:

$$
m_{12 P C R 2}\left(\theta_{1}\right)=0.58 ; \quad m_{12 P C R 2}\left(\theta_{2}\right)=0.42 ;
$$

Let's now combine $m_{12 P C R 2}($.$) with the bba of the third source m_{3}($.$) :$

Then, after applying PCR2 to the obtained conjunctive consensus, the final probability mass vector becomes:

$$
m_{12,3 P C R 2}\left(\theta_{1}\right)=0.408 ; \quad m_{12,3 P C R 2}\left(\theta_{2}\right)=0.592 ;
$$

From this result it is evident, that the final bba $m_{12,3 P C R 2}()=.\left[\begin{array}{ll}0.408 & 0.592\end{array}\right]$ starts to reflect the majority opinion, it means that $m_{12,3 P C R 2}\left(\theta_{1}\right)<m_{12,3 P C R 2}\left(\theta_{2}\right)$. If fourth source is considered with a probability mass vector, supporting the majority opinion, i.e. $m_{4}()=.\left\{m_{4}\left(\theta_{1}\right)=0.3 ; \quad m_{4}\left(\theta_{2}\right)=0.7\right\}$, the final probability mass vector becomes:

$$
m_{(12,3), 4 P C R 2}\left(\theta_{1}\right)=0.286 ; \quad m_{(12,3), 4 P C R 2}\left(\theta_{2}\right)=0.714 ;
$$

The new fused vector $m_{(12,3), 4 P C R 2}()=.\left[\begin{array}{ll}0.286 & 0.714\end{array}\right]$ reflects the majority opinion, since $m_{(12,3), 4 P C R 2}\left(\theta_{1}\right)$ decreases more and more and in the same time $m_{(12,3), 4 P C R 2}\left(\theta_{2}\right)$ increases in the same manner.

The comparative results are given in the Table 7 . 


\begin{tabular}{|c|c|}
\hline Ordinary Conjunctive Rule with PCR2 & TCN Rule with fuzzy based PCR2 \\
\hline$m_{(12,3), 4 P C R 2}\left(\theta_{1}\right)=0.286$ & $\tilde{m}_{(12,3), 4 P C R 2}\left(\theta_{1}\right)=0.348$ \\
\hline$m_{(12,3), 4 P C R 2}\left(\theta_{2}\right)=0.714$ & $\tilde{m}_{(12,3), 4 P C R 2}\left(\theta_{2}\right)=0.652$ \\
\hline
\end{tabular}

Table 7: Comparative Results

The new TCN combination rule with fuzzy based PCR2 reflects the majority opinion slowly than PCR2.

\subsection{Example 7 (neutrality of VBA)}

Let's consider a case with the frame of the problem $\theta=\left\{\theta_{1}, \theta_{2}\right\}$ and two independent sources of information:

$$
\left.\begin{array}{l}
m_{1}(.)=\left\{m_{1}\left(\theta_{1}\right)=0.4 ; \quad m_{1}\left(\theta_{2}\right)=0.5 \quad m_{1}\left(\theta_{1} \cup \theta_{2}\right)=0.1\right. \\
m_{2}(.)=\left\{m_{2}\left(\theta_{1}\right)=0.0 ; \quad m_{2}\left(\theta_{2}\right)=0.0 \quad m_{2}\left(\theta_{1} \cup \theta_{2}\right)=1.0\right.
\end{array}\right\}
$$

The second source is characterized with vacuous gbba.

The TCN rule yields the following result:

$$
\tilde{m}(.)=\left\{\tilde{m}\left(\theta_{1}\right)=0.4 ; \quad \tilde{m}\left(\theta_{2}\right)=0.5 ; \quad \tilde{m}\left(\theta_{1} \cup \theta_{2}\right)=0.1\right\}
$$

From the result obtained, it is evident that TCN rule satisfies the principle of neutrality of the vacuous belief assignment (VBA). The min T-norm operator will yield always a result, which is equal to the non-vacuous bba $m_{1}($.$) , because what-$ ever it is, the probability masses, assigned to its corresponding propositions will be always lower or equal than the probability mass, assigned to the full ignorance in $m_{2}()=.m_{2}\left(\theta_{1} \cup \theta_{2}\right)=1.0$. It means that, according to the way of obtaining the degree of association between the focal elements in $m_{1}($.$) , and m_{2}(),.(\tilde{m}(X)=$ $\left.\min \left\{m_{1}\left(X_{i}\right), m_{2}\left(X_{j}\right)\right\}\right)$, the resulting bba will become equal to the non-vacuous $m_{1}($.$) .$

\subsection{Main properties of TCN Rule of Combination}

Although TCN Rule is not associative (like most of fusion rules but Dempster's rule and conjunctive rule on free-DSm model), it presents the following advantages:

* The rule is simple and very easy to implement;

* It reflects the majority opinion; 
* The rule is convergent toward idempotence in cases when there are no intersections and unions between the elementary hypotheses;

* It reflects the effect of neutrality of vacuous belief assignment;

* It leads to an adequate solutions in case of total conflict between the sources of information

\section{Conclusions}

In this paper a particular combination rule (TCN rule of combination) based on fuzzy T-conorm/T-norm operators is proposed and analysed. It does not belong to the general Weigthed Operator Class. It overcomes the main limitations of Dempster's rule related to the normalization in case of high conflict and yielding counter-intuitive fusion results. The nice features of the new rule could be defined as: very easy to implement, satisfying the impact of neutral Vacuous Belief Assignment; commutative, convergent to idempotence, reflects majority opinion, assures adequate data processing in case of partial and total conflict between the information granules. It is appropriate for the needs of temporal multiple target tracking. The general drawback of this rule is related to the lack of associativity, which is not a main issue in temporal data fusion applications, as those involved in target type tracking [2] and classification.

\section{Acknowlegement}

This work is partially supported by the Bulgarian National Science Fund- grants MI1506/05, EC FP6 funded project - BIS21++ (FP6-2004-ACC-SSA-2)

\section{References}

[1] Blackman S., "Multitarget tracking with Radar Apllications", Artech House, 1986.

[2] Dezert J., A.Tchamova, F. Smarandache, P. Konstantinova, "A Comparison Analysis of Fusion Rules for Target Type Tracking", to appear in Advances and Applications of DSmT for Information Fusion, (Collected Works, Vol. 2), F. Smarandache and J. Dezert (Editors), American Research Press (in preparation), July 2006.

[3] Mendel J.M., "Fuzzy Logic Systems for Engineering: A Tutorial", Proc. of the IEEE, Vol. 83, No.3, pp. 345-377, March 1995.

[4] Shafer G., "A Mathematical Theory of Evidence", Princeton University Press, Princeton, NJ, 1976. 
[5] Smarandache F., J. Dezert (Editors), "Advances and Applications of DSmT", American Research Press, Rehoboth, Vol.1, 2004.

[6] Smarandache F., "Unification of Fusion Theories (UFT)", NATO Advance Study Institute Albena, Bulgaria, 16-27 May, 2005.

[7] Smarandache F., Dezert J., Information Fusion Based on New Proportional Conflict Redistribution Rules, Proceedings of Fusion 2005 International Conference on Information Fusion, Philadelphia, PA, July 26-29,2005.

[8] Smarandache F., Dezert J., "Proportional Conflict Redistribution Rules for Information Fusion", to appear in Advances and Applications of DSmT for Information Fusion, (Collected Works, Vol. 2), F. Smarandache and J. Dezert (Editors), American Research Press (in preparation), July 2006 (preliminary version available at http://arxiv.org/pdf/cs.AI/0408064).

[9] Zadeh L., "On the validity of Dempster's rule of combination", Memo M 79/24, Univ. of California, Berkeley, 1979.

[10] Zadeh L., "A simple view of the Dempster-Shafer theory of evidence and its implication for the rule of combination", AI Magazine 7, No.2, pp. 85-90, 1986.

\begin{abstract}
ALBENA TCHAMOVA is an Associate Professor at the Department of Mathematical Methods for Sensor Information Processing, Institute for Parallel Processing, Bulgarian Academy of Sciences. She received M.Sc. degree in Microelectronics and Ph.D. degree in Radiolocation and Radionavigation (Target Attributes Identification and Classification on the base of Evidence Reasoning Theory and Fuzzy Logic) from the Technical University of Sofia, Bulgaria, in 1978 and 1998 respectively. She worked as a researcher in numerous academic and industry projects (Bulgarian and international) in the area of Multisensor Multitarget Tracking (MS-MTT) and Data Fusion. She is a member of the IEEE, member of International Society for Information Fusion (ISIF), Association of European Operational Research Societies on Fuzzy Sets (EUROFUSE), Image Recognition Society, BAS. Dr. Tchamovas current research interests include MS-MTT, Target Identification, Dezert-Smarandache Theory of Plausible and Paradoxical Reasoning, Information Fusion, Decision Making under Uncertainty. E-mail: tchamova@bas . bg.
\end{abstract}

JEAN DEZERT see his biography in page 44.

FLORENTIN SMARANDACHE see his biography in page 44. 